Algebraic 83 Geometric $\mathcal{T}$ opology

Volume 5 (2005) 129-134

Published: 10 March 2005

ATG

\title{
Regular geodesic languages and the falsification by fellow traveler property
}

\author{
MurRAy ELDER
}

\begin{abstract}
We furnish an example of a finite generating set for a group that does not enjoy the falsification by fellow traveler property, while the full language of geodesics is regular.
\end{abstract}

AMS Classification 20F65; 20F10, 68Q80

Keywords Regular language, falsification by fellow traveler property

\section{Introduction}

In this short note we answer the following question of Neumann and Shapiro from [5]:

Question Can one find a monoid generating set $A$ of a group $G$ so that the language of geodesics is regular but $A$ does not have the falsification by fellow traveler property?

The converse to this statement is Proposition 4.1 of their paper, which states that if $A$ has the falsification by fellow traveler property then the full language of geodesics on $A$ is regular, and this fact is the reason for the property's existence. Several authors have used the falsification by fellow traveler property as a route to finding other (geometric) properties of groups; Rebbechi uses a version of the property to prove that relatively hyperbolic groups are biautomatic [6], and the author exploits the property to prove that a certain class of groups is almost convex [4]. The author discusses various attributes and extensions of the property in [1, 2, 3].

In this article we answer the question via an example first given by Cannon to demonstrate that a group may have a regular language of geodesics with respect to one generating set but not another. Neumann and Shapiro include it in [5] to prove that the falsification by fellow traveler property is generating set dependent. 
The author wishes to thank Walter Neumann and Jon McCammond for ideas and help with this paper.

\section{Definitions}

Definition 2.1 (Finite state automaton; regular language) Let $A$ be a finite set of letters, and let $A^{*}$ be the set of all finite strings, including the empty string, that can be formed from the letters of $A$. A finite state automaton is a quintuple $\left(S, A, \tau, Y, s_{0}\right)$, where $S$ is a finite set of states, $\tau$ is a map $\tau: S \times A \rightarrow S, Y \subseteq S$ are the accept states, and $s_{0} \in S$ is the start state. A finite string $w \in A^{*}$ is accepted by the finite state automaton if starting in the state $s_{0}$ and changing states according to the letters of $w$ and the map $\tau$, the final state is in $Y$. The set of all finite strings that are accepted by a finite state automaton is called the language of the automaton. A language $L \subseteq A^{*}$ is regular if it is the language of a finite state automaton.

Suppose $G$ is a group with finite generating set $A$. A word in $A^{*}$ represents a path in the Cayley graph based at any vertex. Define $d(a, b)$ to be the distance between two points $a$ and $b$ in the Cayley graph with respect to the path metric. Paths can be parameterized by non-negative $t \in \mathbb{R}$ by defining $w(t)$ as the point at distance $t$ along the path $w$ if $t$ is between 0 and the length of $w$, and the endpoint of $w$ otherwise.

Definition 2.2 (The (asynchronous) fellow traveler property) Paths $u$ and $v$ are said to $k$-fellow travel if $d(u(t), v(t)) \leq k$ for all $t \geq 0$. They asynchronously $k$-fellow travel if there is a non-decreasing proper continuous function $\phi:[0, \infty) \rightarrow[0, \infty)$ such that $d(u(t), v(\phi(t))) \leq k$. A language $L \subseteq A^{*}$ enjoys the (asynchronous) fellow traveler property if there is a constant $k$ such that for each $u, v \in L$ that start at the identity and end at distance 0 or 1 apart in the Cayley graph, $u$ and $v$ (asynchronously) $k$-fellow travel.

Definition 2.3 (The (asynchronous) falsification by fellow traveler property) A finite generating set $A$ for a group $G$ has the (asynchronous) falsification by fellow traveler property if there is a constant $k$ such that every non-geodesic word in the Cayley graph of $G$ with respect to $A$ is (asynchronously) $k$-fellow traveled by a shorter word.

The property arises naturally in the context of geodesic regular languages, and the proof of Proposition 4.1 in [5] uses the property to build an appropriate 
finite state automaton. The author proves in [1] that the synchronous and asynchronous versions of the falsification by fellow traveler property are equivalent.

\section{The Example}

Let $G$ be the split extension of $\mathbb{Z}^{2}$, generated by $\{a, b\}$, by $\mathbb{Z}_{2}$, generated by $\{t\}$, such that $t$ conjugates $a$ to $b$ and $b$ to $a$, with presentation

$$
\left\langle a, b, t \mid t^{2}=1, a b=b a, t a t=b\right\rangle .
$$

Performing one Tietze transformation (removing $b=t a t$ ) we obtain

$$
\left.\langle a, t| t^{2}=1, \text { atat }=\text { tata }\right\rangle .
$$

Let $A=\left\{a^{ \pm 1}, t^{ \pm 1}\right\}$ be the inverse-closed generating set corresponding to this presentation. The Cayley graph for $G$ with respect to $A$ is shown in Figure 1. We can consider the vertices of the graph as either being in the top or the

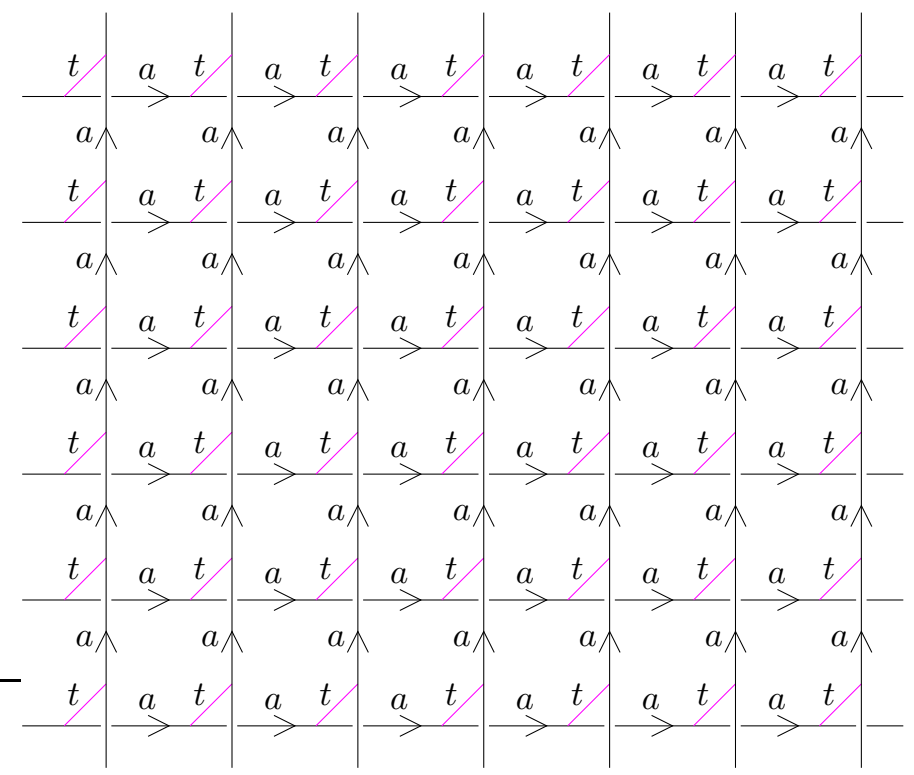

Figure 1: The Cayley graph for $G=\langle a, t| t^{2}=1$, atat $=$ tata $\rangle$

bottom layer, where edges labeled $t$ link top and bottom layers. We declare the identity vertex to lie in the bottom. Each vertex can also be given a coordinate $(x, y)$ where $x$ is the distance in the East-West direction from the identity, and 
$y$ is the distance in the North-South direction. In this way each vertex (group element) is uniquely specified by the triple $(x, y$, bottom) or $(x, y$, top $)$.

For example, the identity has the coordinate $\left(0,0\right.$, bottom), the word $a^{3} t a^{4}$ has the coordinate $(3,4$, top $)$, the word $a^{3} t a^{4} t$ has the coordinate $(3,4$, bottom), and the word $t a^{3} t a^{4}$ has the coordinate (4,3, bottom).

Lemma 3.1 Each word from 1 to a vertex in the top layer has an odd number of $t$ letters, and each word from 1 to a vertex in the bottom layer has an even number of $t$ letters.

Proof Suppose a vertex has the coordinate $\left(x, y\right.$, top). Then $a^{x} t a^{y}$ is a word to this vertex. Suppose $w$ is any other word to this vertex. Then $w a^{-y} t a^{-x}={ }_{G} 1$ so under the map which sends $t$ to $t$ and $a$ to 1 this word must be sent to an even power of $t$, so $w$ has an odd number of $t$ letters. Similarly if a vertex lies in the bottom layer there is a word $a^{x} t a^{y} t$ to it from 1 . If $w$ is any other word to this vertex then $w t a^{-y} t a^{-x}={ }_{G} 1$ gets sent to an even power of $t$ so $w$ has an even number of $t$ letters.

Lemma 3.2 The word $t a^{n} t a^{m} t$ for any $m, n \in \mathbb{Z}$ is not geodesic.

Proof The word $t a^{n} t a^{m} t$ can be written as $a^{m} t a^{n}$ which is shorter.

Lemma 3.3 Each vertex in the top layer has a unique geodesic to it from the identity of the form $a^{x} t a^{y}$, where $(x, y$, top) is the coordinate of the vertex.

Proof By Lemma 3.1 any geodesic to the vertex with coordinate $(x, y$, top) has an odd number of $t$ letters. If a word has three or more $t$ letters then it has a subword of the form $t a^{n} t a^{m} t$ which is not geodesic by Lemma 3.2. So any geodesic to this vertex has exactly one $t$ letter, so is of the form $a^{i} t a^{j}$. This path has coordinate $(i, j$, top) so it must be that $i=x$ and $j=y$.

Proposition 3.4 A does not have the falsification by fellow traveler property.

Proof Suppose by way of contradiction that $A$ has the falsification by fellow traveler property with positive constant $k$, and choose $n>>k$. Consider the word $w=t a^{n} t a^{n} t$ which ends at the coordinate $(n, n$, top). Any word that ends at this vertex must move East at least $n$ units and North at least $n$ units, and by Lemma 3.1 must have an odd number of $t$ letters. If it has just one $t$ letter then it must be the unique geodesic $a^{n} t a^{n}$ which clearly does not $k$-fellow travel $w$. Otherwise it has three or more $t$ letters, so has length at least $2 n+3$ so is not shorter than $w$, so we are done. 
Theorem 3.5 There is a group and finite generating set such that the language of all geodesics is regular but fails to have the falsification by fellow traveler property.

Proof By Proposition 3.4 the group $G$ with generating set $A$ fails the falsification by fellow traveler property.

Consider the language $L=\left\{a^{x}, a^{x} t a^{y}, a^{x_{1}} t a^{y} t a^{x_{2}}: x, x_{1}, x_{2}, y \in \mathbb{Z}, x_{1} \cdot x_{2} \geq 0\right\}$. $L$ is the language of the finite state automaton in Figure 2, All states are accept states.

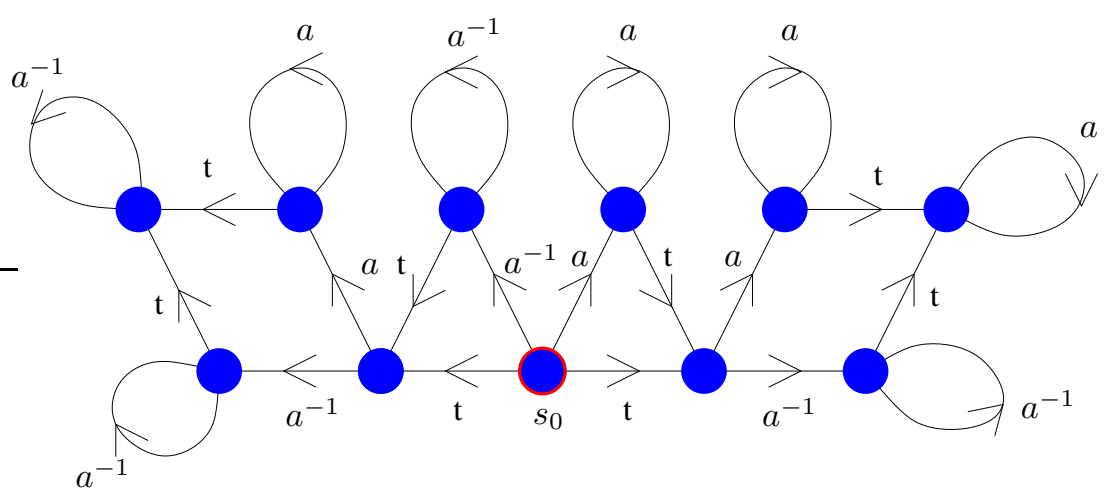

Figure 2: A finite state automaton accepting the language $L$

We now show that $L$ is the language of all geodesics on $A$ for $G$. By Lemma 3.3 every group element corresponding to a vertex in the top layer has a unique geodesic representative of the form $a^{x} t a^{y}$. Otherwise the group element corresponds to a vertex in the bottom, so has an even number of $t$ letters. If a word has more than two $t$ letters then it has a subword of the form $t a^{n} t a^{m} t$ which is not geodesic by Lemma 3.2. so a geodesic word for a bottom element has either zero $t$ letters, so is of the form $a^{x}$, or has two $t$ letters, so is of the form $a^{x_{1}} t a^{y} t a^{x_{2}}$. If $x_{1}$ and $x_{2}$ don't have the same sign then we can find a shorter word $a^{\left(x_{1}+x_{2}\right)} t a^{y} t$. Otherwise $a^{x_{1}} t a^{y} t a^{x_{2}}$ is a geodesic to a vertex with coordinate $\left(x_{1}+x_{2}, y\right.$, bottom). Notice that this gives a family of $x_{1}+x_{2}+1$ geodesics to this vertex.

\section{References}

[1] Murray J Elder, Finiteness and the falsification by fellow traveler property, Geom. Dedicata 95 (2002) 103-113 MathReview 
[2] Murray J Elder, The loop shortening property and almost convexity, Geom. Dedicata 102 (2003) 1-18 MathReview

[3] Murray J Elder, Patterns theory and geodesic automatic structure for a class of groups, Internat. J. Algebra Comput. 13 (2003) 203-230 MathReview

[4] Murray J Elder, A non-Hopfian almost convex group, J. Algebra 271 (2004) 11-21 MathReview

[5] Walter D Neumann, Michael Shapiro, Automatic structures, rational growth, and geometrically finite hyperbolic groups, Invent. Math. 120 (1995) 259-287 MathReview

[6] Donovan Rebbechi, Algorithmic Properties of Relatively Hyperbolic Groups, PhD Dissertation, Rutgers Newark, arXiv:math.GR/0302245

School of Mathematics and Statistics, University of St Andrews

North Haugh, St Andrews, Fife, KY16 9SS, Scotland

Email: murray@mcs.st-and.ac.uk

URL: http://www-groups.mcs.st-andrews.ac.uk/ murray/

Received: 21 September 2004 